\title{
Cardiac Conductivity Values - A Challenge for Experimentalists?
}

\author{
Peter R. Johnston \\ School of Biomolecular and Physical Sciences \\ and Queensland Micro- and Nanotechnology Centre \\ Griffith University, Nathan, QLD, Australia, 4111. \\ Email: P.Johnston@griffith.edu.au
}

\begin{abstract}
Recent experimental studies have challenged the usual assumption that cardiac conductivities in the direction normal to the fibres but in the plane of the fibre sheet are equal to those in the direction normal to the sheet. Here, a simulation study, using a simple model of cardiac tissue, is undertaken to consider the effect that this experimental observation has on the resulting epicardial potential distribution. Results indicate that there is a significant difference when using six conductivity parameters as opposed to the usual four values, where the approximation of equality of the above mentioned conductivities is made. The results are compared with experimentally observed epicardial potential distributions and an explanation of the results is offered in terms of voltage dividers. It is concluded that further experimental studies are required to obtain reliable conductivity values which can be used in simulation models.
\end{abstract}

\section{INTRODUCTION}

Many simulation studies have shown the importance of cardiac conductivity values in simulating aspects of the electrocardiogram. In particular, several studies [1]-[4] have focussed on how the conductivity values affect the shape of the epicardial potential distribution in simple models of partial thickness ischaemia in the heart during the ST segment. The goal of these studies has been to compare simulated epicardial potential distributions with those obtained from experimental measurements in the sheep model [5].

Based on the results of these experimental studies in the sheep [5], simulation studies should be able to reproduce two key features of the epicardial potential distribution. Firstly, large potential gradients should occur near the ischaemic boundaries and, secondly, there should be some degree of ST elevation present above the ischaemic region, especially as the region of ischaemia becomes thicker.

The electric field arising from partial thickness ischaemia in cardiac tissue is governed by the passive bidomain equation [4] and, in complete generality, contains six conductivity values. These are intracellular $(i)$ and extracellular $(e)$ conductivities in the longitudinal $(l)$ (along the fibres), transverse $(t)$ (normal to the fibre direction, but within the fibre sheet) and normal ( $n$ ) (normal to the fibre sheets) directions. Most simulation studies to date have made the assumption that the conductivity in the transverse direction is the same as the conductivity in the normal direction.

However, recent experimental studies have shown that this is not the case [6], [7]. These studies indicate that the ratio of total (the sum of intra- and extracellular) longitudinal to transverse to normal conductivities is $4: 2: 1$. The implication here is that six conductivity values should be used in simulation studies.

One recent simulation study [8] has used six conductivity values, all based on general dielectric properties of the cardiac tissue [9]. This study, performed in a whole torso model, shows that ST depression should occur above the region of subendocardial ischaemia, in contrast to the experimental observations of Li et al. [5].

The present study will compare the differences between the use of four or six conductivity values in a simple slab model of cardiac tissue. Firstly, a survey of the assumption of equal transverse and normal conductivity parameters will be considered in the context of experimental results. Then, this assumption will be relaxed in order to study the effect of six conductivity parameters. These results will be compared to the relevant four parameter simulations and to the experimental results. An explanation for the results in terms of voltage dividers will also be offered.

\section{Methods}

\section{A. Governing Equations}

During the ST segment the electric field in the heart is assumed to be stationary. Hence the electric potential in the cardiac tissue is governed by the steady state passive bidomain equation [4]

$$
\nabla \cdot\left(\mathbf{M}_{i}+\mathbf{M}_{e}\right) \nabla \phi_{e}=-\nabla \cdot \mathbf{M}_{i} \nabla \phi_{m}
$$

where $\phi_{e}$ is the extracellular potential in the cardiac tissue, $\phi_{m}$ is the specified transmembrane potential distribution and $\mathbf{M}_{i}$ and $\mathbf{M}_{e}$ represent conductivity tensors for the intracellular $(i)$ and extracellular $(e)$ spaces, respectively. These conductivity tensors contain information about the local fibre direction.

The conductivity tensors take the form [10]

$$
\mathbf{M}_{h}=\mathbf{A G}_{h} \mathbf{A}^{T}
$$

for $h=i$ or $e$, where $\mathbf{G}_{h}$ is a diagonal matrix containing the conductivity values along the fibre direction, $g_{h l}$, across the sheets of fibres, $g_{h t}$, and between the sheets of fibres, $g_{h n}$. The matrix $\mathbf{A}=\left\{a_{i j}\right\}$ is a rotation matrix mapping the local 

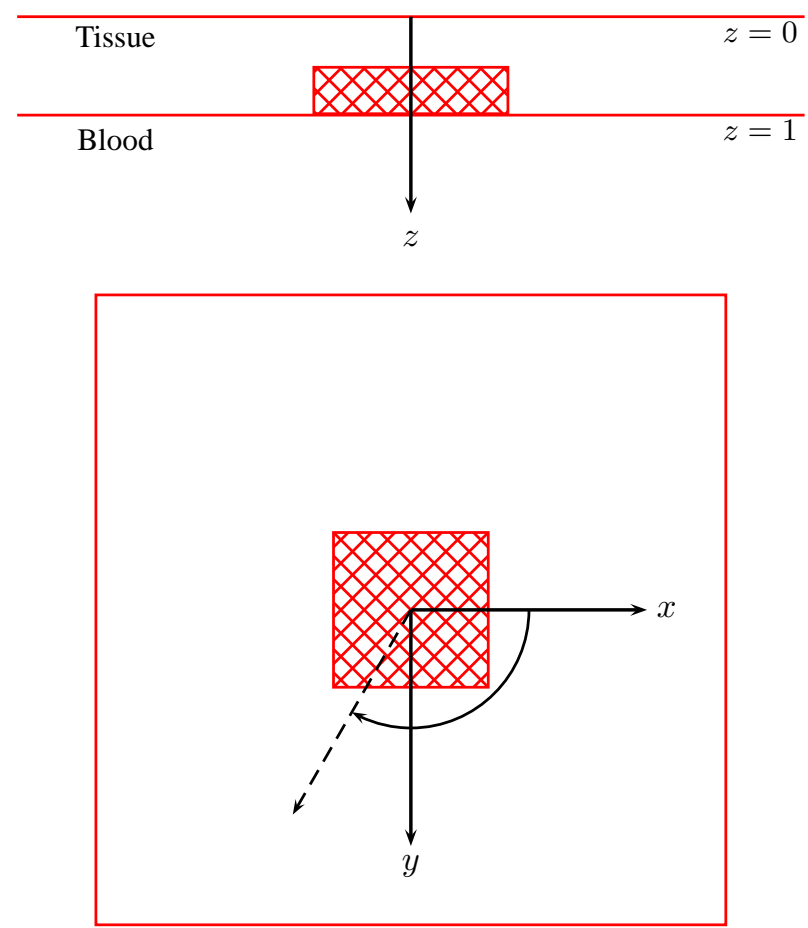

Fig. 1. A schematic view of the tissue-blood model used in this study. The top panel is a cross-section view of the tissue $(0 \leq z \leq 1)$ and the blood $(z>1)$ with the positive $z$ axis pointing downwards. The hashed region indicates ischaemic tissue. The bottom panel is a plan view of the model, looking from the $-z$ direction, again with the hashed region indicating the ischaemic tissue The dashed arrow indicates the fibre direction on the endocardium (at $z=1$ ) when the fibre direction on the epicardium (at $z=0$ ) points along the $x$-axis

fibre direction into the global coordinate system. Under these assumptions it can be shown that

$$
\mathbf{M}_{h}=g_{h t} \mathbf{I}+\left(g_{h l}-g_{h t}\right) \hat{\mathbf{a}}_{\mathbf{l}}^{T} \hat{\mathbf{a}}_{\mathbf{l}}+\left(g_{h n}-g_{h t}\right) \hat{\mathbf{a}}_{\mathbf{n}}^{T} \hat{\mathbf{a}}_{\mathbf{n}}
$$

where $\mathbf{I}$ is the $3 \times 3$ identity matrix, $\hat{\mathbf{a}}_{\mathbf{l}}$ is the unit vector indicating the local direction of the fibres [11] and $\hat{\mathbf{a}}_{\mathbf{n}}$ is the unit vector normal to the fibre sheet.

It will be further assumed that the cardiac tissue is in contact with a blood mass. The electric potential in the blood mass, $\phi_{b}$, is governed by Laplace's equation

$$
\nabla^{2} \phi_{b}=0
$$

\section{B. Model Geometry}

For the purposes of this study it will be assumed that the computational domain is represented by a finite slab of cardiac tissue with fibres on the epicardial surface parallel to the $x$ axis. The origin of the rectangular $(x, y, z)$ coordinate system is placed in the centre of the tissue slab with the epicardial surface represented by the $x-y$ plane (as shown in Figure 1). It will be further assumed that the cardiac tissue is of unit thickness and is attached to a blood mass at the endocardial surface at $z=1$ which is of finite size in the $z$-direction such that it is much larger than the thickness of the cardiac tissue.

Placed at the centre of the slab of tissue is a region of partial thickness ischaemia extending from the endocardium towards the epicardium. This region of ischaemia is parallelepipedal in shape with sides aligned with the $x$ and $y$ coordinate axes.

The transition between the normal and ischaemic tissue is described by a smooth variation in the transmembrane potential during the ST segment. The difference in plateau transmembrane potential between normal and ischaemic tissue, $\Delta \phi_{p}$, is set at $-30 \mathrm{mV}$ and the transition is described in terms of exponential and hyperbolic functions [1], [12].

Fibres in the tissue will be assumed to rotate through an angle of $120^{\circ}$ (chosen for consistency with previous models [1], [2]). If the fibres on the epicardium are aligned along the positive $x$-axis, then the longitudinal direction at any depth $z$, with respect to the positive $x$-axis is given by

$$
g(z)=\frac{2 \pi}{3} z .
$$

As mentioned above, this rotation is taken into account in the conductivity tensors by assuming that

$$
\mathbf{A}=\left(\begin{array}{ccc}
\cos g(z) & \sin g(z) & 0 \\
-\sin g(z) & \cos g(z) & 0 \\
0 & 0 & 1
\end{array}\right)
$$

\section{Boundary Conditions}

To solve the governing equations, several boundary conditions are required. It will be assumed that the epicardial surface is insulated, i.e.

$$
\text { at } z=0, \quad \frac{\partial \phi_{e}}{\partial z}=0
$$

It will also be assumed that the edges of the tissue and the blood mass in the $x$ and $y$ directions are insulated, i.e.

$$
\begin{aligned}
\left(\mathbf{M}_{i}+\mathbf{M}_{e}\right) \nabla \phi_{e} \cdot \mathbf{n}=0 & \text { for } \quad 0 \leq z \leq 1 \\
\nabla \phi_{b} \cdot \mathbf{n}=0 & \text { for } \quad z \geq 1
\end{aligned}
$$

where $\mathbf{n}$ is the appropriate outward pointing normal. At the bottom of the blood mass the potential is set to zero. Finally, continuity of potential and current is assumed between the tissue and the blood mass, so at $z=1$

$$
\phi_{e}=\phi_{b} \quad \text { and } \quad g_{e t} \frac{\partial \phi_{e}}{\partial z}=g_{b} \frac{\partial \phi_{b}}{\partial z}
$$

where $g_{b}$ is the conductivity of blood, set at $6.7 \mathrm{mS} / \mathrm{cm} \mathrm{[13].}$ Given the scale of the problem, this is an appropriate boundary condition to use [14].

\section{RESULTS}

For this study, a slab of cardiac tissue of size $16 \mathrm{~cm} \times 16 \mathrm{~cm}$ in the $x$ and $y$ directions and of thickness $1 \mathrm{~cm}$, as described previously [1], was used. The governing equations were solved using the finite volume method [15] in which the blood mass had a depth of $26 \mathrm{~cm}$, the bottom face of which was set as the zero of potential. The centrally located ischaemic region had a size of $4 \mathrm{~cm} \times 4 \mathrm{~cm}$ in the $x$ and $y$ directions and covered $50 \%$ of the depth of the tissue. All ischaemic borders were set at a value of 0.01 , leading to a sharp interface between normal and ischaemic tissue. Finally, as mentioned above, fibre rotation was set at $120^{\circ}$ [16]. 
TABLE I

CONDUCTIVITY DATA FROM THE INDICATED STUDIES (GIVEN IN MS/CM). (THE DASH (-) INDICATES THAT THE CORRESPONDING VALUES ARE NOT AVAILABLE FOR THAT DATA SET.)

\begin{tabular}{|l|c|c|c|c|c|c|}
\hline Study & $g_{i l}$ & $g_{e l}$ & $g_{i t}$ & $g_{e t}$ & $g_{i n}$ & $g_{e n}$ \\
\hline Clerc [17] & 1.7 & 6.2 & 0.19 & 2.4 & - & - \\
$\begin{array}{l}\text { Roberts et } \\
\text { al. [18] }\end{array}$ & 2.8 & 2.2 & 0.26 & 1.3 & - & - \\
$\begin{array}{l}\text { Roberts \& } \\
\text { Scher [19] }\end{array}$ & 3.4 & 1.2 & 0.6 & 0.8 & - & - \\
$\begin{array}{l}\text { Hooks [7] } \\
\text { MacLachlan }\end{array}$ & 2.63 & 2.63 & 0.263 & 2.45 & 0.08 & 1.087 \\
et al. [8] & 2.0 & 1.0 & 1.65 & 0.31525 & 1.3514 \\
\hline
\end{tabular}

The conductivity parameters used in the study are summarised in Table I. For consistency with previous studies, the conductivity data from Clerc [17], Roberts et al. [18] and Roberts and Scher [19] are included. These data contain only four conductivity values because of the experimental procedure used to determine the values. Also included is a recent set of six conductivity values from Hooks [7] and the six conductivity values used by MacLachlan et al. [8], which were based on the work of Foster and Schwan [9].

Fig. 2 shows epicardial potential distributions on the surface of the slab of cardiac tissue, based on the conductivity data of Clerc (panel (a)), Roberts et al. (panel (b)) and Roberts and Scher $(\operatorname{panel}(\mathrm{c}))$. These distributions have been presented previously [2] and discussed at length. Summarising briefly, the data of Clerc and Roberts et al. show three potential wells on the surface, with large potential gradients between the wells. However, the data of Roberts and Scher show a single potential well with no large potential gradients. None of the distributions shows any increase in potential near the ischaemic region.

Fig. 3 shows the epicardial potential distributions based on the six conductivity values of Hooks (panel (a)) and MacLachlan et al. (panel (b)). The distribution obtained from the data of Hooks shows large potential gradients above the ischaemic boundary, in keeping with the distributions obtained from the data of Clerc (Fig. 2(a)) and Roberts et al. (Fig. 2(b)). However, in contrast to these distributions, the data of Hooks give rise to a central region of positive potential. The data of MacLachlan et al. also show three wells of potential as well as large potential gradients, very similar to the distributions from the data of Clerc and Roberts et al. However, no positive potentials are observed.

Finally, Fig. 4 shows the epicardial potential distributions when the approximation $g_{i t}=g_{i n}$ and $g_{e t}=g_{e n}$ is enforced for the data of Hooks (panel (a)) and MacLachlan et al. (panel (b)). It can be seen here that for the data of Hooks, the large region of positive potential has almost disappeared, being replaced with two spikes and a larger central well. The large potential gradients remain. In the case of the data from MacLachlan et al., the three potential wells observed with six conductivity values have merged into one and the distribution is very similar to that obtained from the data of Roberts and Scher (Fig. 2(c)).

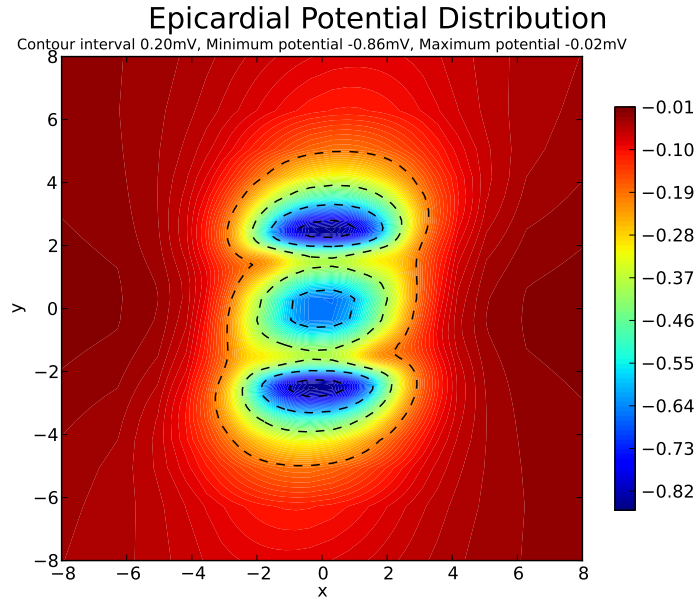

(a) Clerc [17]

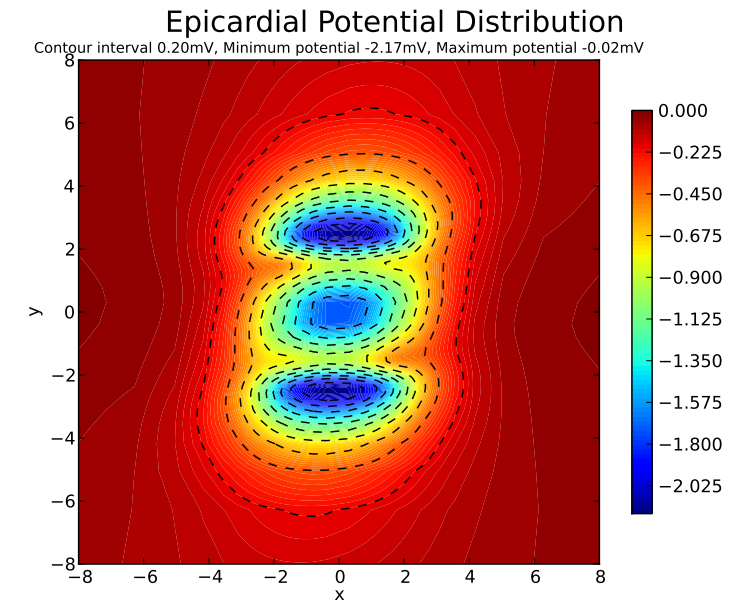

(b) Roberts et al. [18]

Epicardial Potential Distribution

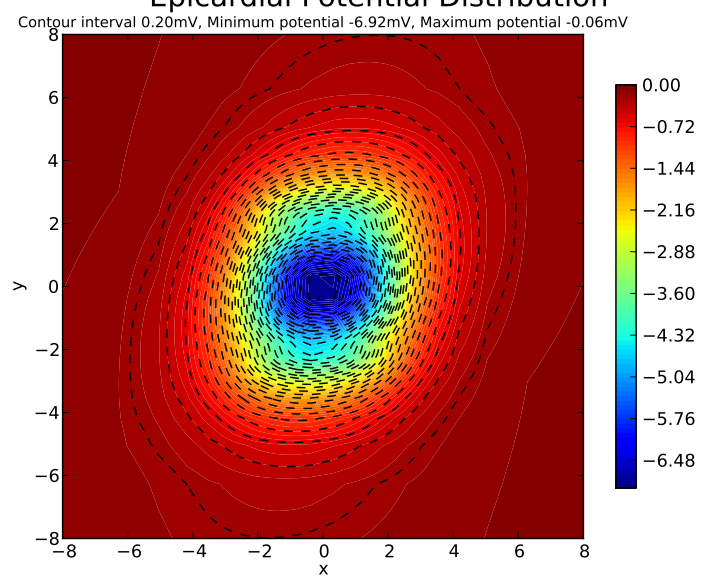

(c) Roberts \& Scher [19]

Fig. 2. Epicardial potential distributions in a slab model of cardiac tissue for three different conductivity data sets, each with four conductivity values. Panel (a) is generated from the data of Clerc [17], panel (b) is generated from the data of Roberts et al. [18] and panel (c) is generated from the data of Roberts and Scher [19]. Negative potentials are indicated by thin dashed lines and positive potentials are indicated by the thin solid line with the zero of potential represented by a thick solid line. 
Epicardial Potential Distribution

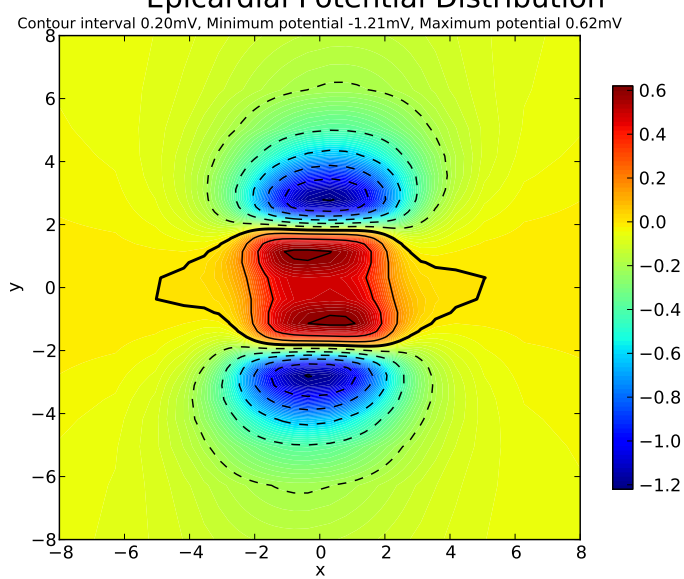

(a) Hooks [7]

Epicardial Potential Distribution

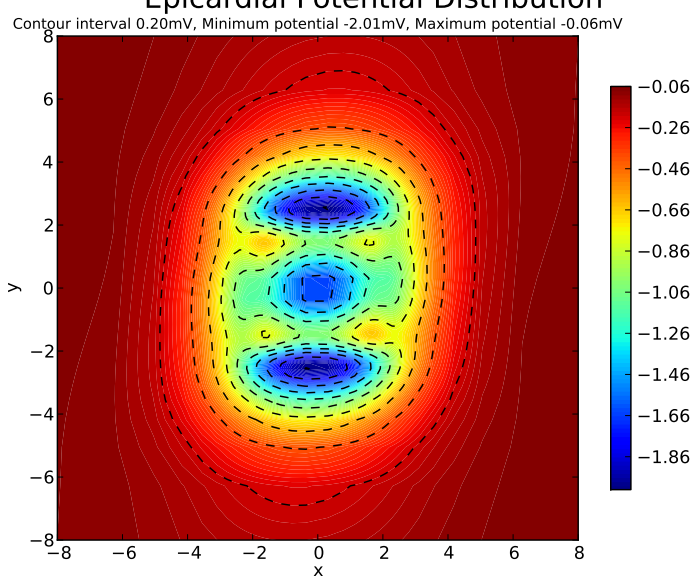

(b) MacLachlan et al. [8]

Fig. 3. Epicardial potential distributions in a slab model of cardiac tissue for two different conductivity data sets, each with six conductivity values. Panel (a) is generated from the data of Hooks [7] and panel (b) is generated from the data of MacLachlan et al. [8]. The format is the same as in Fig. 2.

\section{Discussion}

This study has presented a simulation study comparing epicardial potential distributions obtained using four and six conductivity values. While the results presented in Fig. 2 are not new, they are included to complement the discussion of more recently published data for six conductivity values. The interested reader is directed to reference [2] for a more complete discussion of the significance of these results.

As observed in the previous section, making the approximation $g_{i t}=g_{i n}$ and $g_{e t}=g_{e n}$ induces a significant change in the epicardial potential distributions. The use of six conductivity values can yield a region of positive potential above the region of ischaemia, with high potential gradients at the borders of the ischaemic region, Fig. 3(a). These features appear to match similar observations from experimental studies [5]. However, this is not always the case for six conductivity values, as shown in Fig. 3(b), which only matches the experimental observations by exhibiting large potential

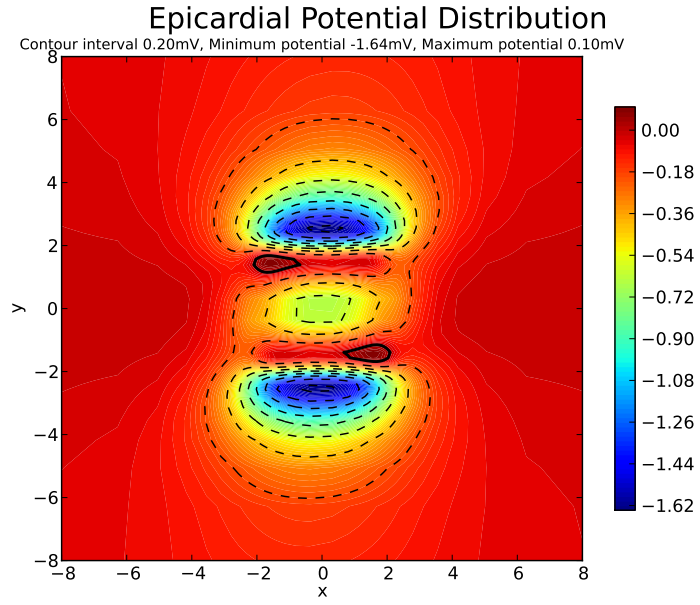

(a) Hooks [7]

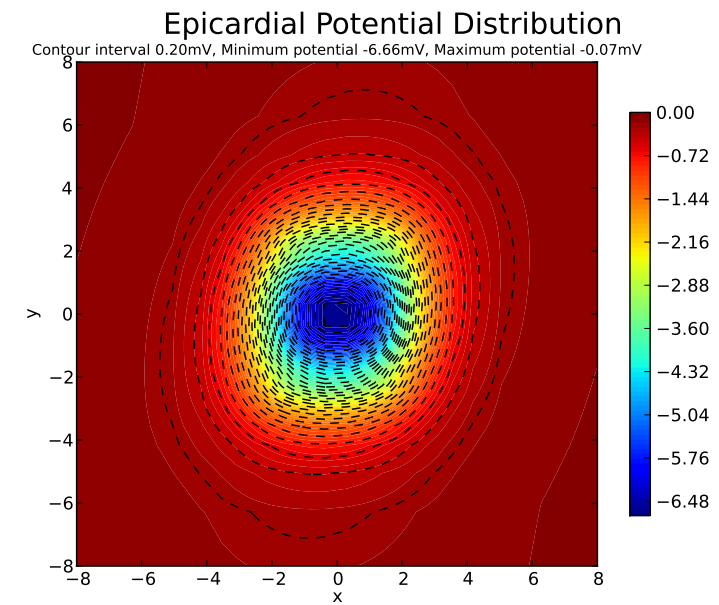

(b) MacLachlan et al. [8]

Fig. 4. Epicardial potential distributions in a slab model of cardiac tissue for two different conductivity data sets, with the approximation $g_{i t}=g_{i n}$ and $g_{\text {et }}=g_{\text {en }}$. Panel (a) is generated from the data of Hooks [7] and panel (b) is generated from the data of MacLachlan et al. [8]. The format is the same as in Fig. 2.

gradients near the ischaemic boundary. Further, using four conductivity values yields only the high potential gradients over the ischaemic boundary, but not the region of relatively positive potential above the ischaemic region, and then only in some cases, Figs. 2(a), 2(b) and 4(a).

It is possible to observe a peak in potential above the ischaemic region in some simulations based on only four conductivity values, as shown in Hopenfeld et al. [4]. The fundamental difference between the simulations presented there and those presented here is that in [4], the ratio $g_{i l} / g_{i t}$ was at least 20 , a value chosen based on previous simulation studies [20]. Here, this ratio is 10 or less, more in keeping with other experimental measurements.

A possible explanation for the observed epicardial potential distributions from the data of Hooks can be given in terms of the voltage divider interpretation presented by Hopenfeld et al. [4], [21]. With four conductivity values (or assuming that $g_{i t}=g_{i n}$ and $g_{e t}=g_{e n}$ ), only two ischaemic boundaries 
are important in determining current loops within the tissue. These are the boundary across the longitudinal direction of the fibres (the lateral boundary) and the boundary at the top of the ischaemic region (the transmural boundary). The current loops generated depend on the relative strengths of the current dipole generated across these two boundaries. There is, however, another boundary to the ischaemic region which is parallel to the direction of the fibres (call it the parallel boundary). Since it is assumed that $g_{i t}=g_{i n}$ and $g_{e t}=g_{e n}$, no current loops are generated between this boundary and the transmural boundary.

When six conductivities are used, current loops are generated between the lateral boundary and the transmural boundary, as well as between the parallel boundary and the transmural boundary. Since we have that $g_{i l}>g_{i t}>g_{i n}$ and $g_{e l}>g_{\text {et }}>g_{\text {en }}$ the current dipoles are stronger than they are in the four conductivity situation and therefore promote a region of greater relatively positive potential above the ischaemic region.

On the other hand, the full argument must be more complex than that given above, as it does not explain the epicardial potential distributions which arise from the data of MacLachlan et al..

A concern which arises is the variability between conductivity values reported. Although the values of Hooks and MacLachlan et al. are similar, they produce quite different epicardial potential distributions. The conductivity values given in MacLachlan et al. come from [9], but the values do not appear explicitly in that manuscript. Further, the six values given in [7] do not not appear to have been measured individually. It appears that the authors chose representative values of $g_{i l}$, $g_{e l}, g_{i t}$ and $g_{e t}$ and then calculated $g_{i n}$ and $g_{e n}$ from a minimisation procedure [6].

\section{CONCLUSIONS}

This study has considered the effect a set of six conductivity values has on epicardial potential distributions arising from subendocardial ischaemia in a slab of cardiac tissue. That is, the often made assumption that $g_{i t}=g_{i n}$ and $g_{e t}=g_{e n}$ has been relaxed. The simulations show that including the extra conductivity values in the direction normal to the plane of the tissue produces epicardial potential distributions which more closely match observed experimental measurements. There is also a disparity between the two sets of six conductivity values, which leads to significant change in the resulting epicardial potential distributions.

Therefore, the challenge remains to find a consistent set of experimentally determined conductivity values which yields realistic results when applied to simple models of cardiac tissue. These values need to be absolute, relative to the conductivity of blood, and obtained for in vivo tissue, which is both normal and ischaemic.

\section{REFERENCES}

[1] P. R. Johnston, D. Kilpatrick, and C. Y. Li, "The importance of anisotropy in modelling ST segment shift in subendocardial ischaemia," IEEE Transactions on Biomedical Engineering, vol. 48, no. 12, pp. 1366-1376, December 2001

[2] P. R. Johnston and D. Kilpatrick, "The effect of conductivity values on ST segment shift in subendocardial ischaemia," IEEE Transactions on Biomedical Engineering, vol. 50, no. 2, pp. 150-158, February 2003.

[3] P. R. Johnston, "A cylindrical model for studying subendocardial ischaemia in the left ventricle," Mathematical Biosciences, vol. 186, no. 1, pp. 43-61, 2003.

[4] B. Hopenfeld, J. G. Stinstra, and R. S. MacLeod, "The effect of conductivity on st-segment epicardial potentials arising from subendocardial ischemia," Annals of Biomedical Engineering, vol. 33, no. 6, pp. 751-763, 062005.

[5] D. Li, C. Y. Li, A. C. Yong, and D. Kilpatrick, "Source of electrocardiographic ST changes in subendocardial ischemia," Circulation Research, vol. 82, pp. 957-970, 1998.

[6] D. A. Hooks, K. A. Tomlinson, S. G. Marsden, I. J. LeGrice, B. H. Smaill, A. J. Pullan, and P. J. Hunter, "Cardiac microstructure: Implications for electrical propagation and defibrillation in the heart," Circulation Research, vol. 91, no. 4, pp. 331-338, 82002.

[7] D. Hooks, "Myocardial segment-specific model generation for simulating the electrical action of the heart," BioMedical Engineering OnLine, vol. 6, no. 1, pp. 21-21, 2007.

[8] M. C. MacLachlan, J. Sundnes, and G. T. Lines, "Simulation of st segment changes during subendocardial ischemia using a realistic 3d cardiac geometry," Biomedical Engineering, IEEE Transactions on, vol. 52, no. 5, pp. 799-807, 2005.

[9] K. R. Foster and H. P. Schwan, "Dielectic properties of tissue and biological materials: A critical review," Critical Reviews in Biomedical Engineering, vol. 17, no. 1, pp. 25-104, 1989.

[10] R. M. Gulrajani, Bioelectricity and Biomagnetism. New York: John Wiley and Sons, 1998

[11] M. Potse, B. Dubé, and A. Vinet, "Cardiac anisotropy in boundaryelement models for the electrocardiogram," Medical \& Biological Engineering \& Computing, vol. 47, no. 7, pp. 719-729, 2009.

[12] L. Tung, "A bi-domain model for describing ischaemic myocardial DC potentials," Ph.D. dissertation, Massachusetts Institute of Technology, June 1978.

[13] S. Rush, J. A. Abildskov, and R. McFee, "Resistivity of body tissues at low frequencies," Circulation Research, vol. 12, pp. 40-50, 1963.

[14] B. J. Roth, "A comparison of two boundary conditions used with the bidomain model of cardiac tissue," Annals of Biomedical Engineering, vol. 19, pp. 669-678, 1991.

[15] P. R. Johnston, "A finite volume method solution for the bidomain equations and their application to modelling cardiac ischaemia," Computer Methods in Biomechanics and Biomedical Engineering, vol. 13, no. 2, pp. 157-170, 2010.

[16] D. D. Streeter, "Gross morphology and fiber geometry of the heart," in Handbook of Physiology, Vol 1, R. M. Berne, Ed. Baltimore, MD: Williams and Williams, 1979, ch. 2: The Cardiovascular System, pp. $61-112$.

[17] L. Clerc, "Directional differences of impulse spread in trabecular muscle from mammalian heart," Journal of Physiology, vol. 255, pp. 335-346, 1976.

[18] D. E. Roberts, L. T. Hersh, and A. M. Scher, "Influence of cardiac fiber orientation on wavefront voltage, conduction velocity and tissue resistivity in the dog," Circ. Res., vol. 44, pp. 701-712, 1979.

[19] D. E. Roberts and A. M. Scher, "Effects of tissue anisotropy on extracellular potential fields in canine myocardium in situ," Circ. Res. vol. 50, pp. 342-351, 1982.

[20] J. Stinstra, B. Hopenfeld, and R. MacLeod, "On the passive cardiac conductivity," Annals of Biomedical Engineering, vol. 33, no. 12, pp. 1743-1751, 2005.

[21] B. Hopenfeld, J. G. Stinstra, and R. S. MacLeod, "Mechanism for ST depression associated with contiguous subendocardial ischaemia," Journal of Cardiovascular Electrophysiology, vol. 15, pp. 1200-1206, 2004. 